\title{
PERMUTATION MONOTONE FUNCTIONS OF RANDOM VECTORS WITH APPLICATIONS IN FINANCIAL AND ACTUARIAL RISK MANAGEMENT
}

\author{
XIAOHU LI,* Stevens Institute of Technology \\ YINPING YOU, ${ }^{* *}$ Huaqiao University
}

\begin{abstract}
In this paper we develop two permutation theorems on argument increasing functions of a multivariate random vector and a real parameter vector. We use the unified approach of our two theorems to provide some important theoretical results on the capital allocation in actuarial science, the deductible and upper limit allocations in insurance policy, and portfolio allocation in financial engineering. Our results successfully improve or extend the corresponding works in the literature.
\end{abstract}

Keywords: Archimedean copula; arrangement increasing; deductible; distortion measure; stochastic order; upper limit; utility function

2010 Mathematics Subject Classification: Primary 91B16; 91B30

Secondary 60E 15

\section{Section}

Risk measure and risk management play central roles in economics and the financial sciences. Financial engineers strive to optimize the portfolio of random risk assets in pursuit of maximized potential profit, while actuaries endeavour to subdivide better the capital to various insurance products so as to minimize the risk of default. In general, they both deal with a random vector $\boldsymbol{X}=\left(X_{1}, \ldots, X_{n}\right)$ representing either the potential profit or potential loss and a real vector $\lambda=\left(\lambda_{1}, \ldots, \lambda_{n}\right)$ concerning the decision policy of the stakeholder. As a stylized decision problem, the allocation of the total capital to related risks or the allocation of wealth to concerned risk assets so as to increase profit or minimize the risk of default has received much attention in recent decades. These types of optimal allocation problem naturally arise in various situations.

In enterprise risk management (ERM), to supervise the behavior of banks and insurance companies, the regulator establishes rules to determine the level of sufficient capital held by financial institutions so that their financial obligations can be met with a high probability when they are due, even in adverse situations. The level of available capital is a key factor that rating agencies use to assess the financial strength of a company. In practice, an important component of one ERM framework is the exercise of capital allocation, that is, the firm reasonably subdivides the total capital across its various constituents (e.g. business lines, types of exposure, territories, or even individual products in a portfolio of insurance policies) in order to reduce the risk of default.

Received 24 July 2013; revision received 3 March 2014.

* Postal address: Department of Mathematical Sciences, Stevens Institute of Technology, Hoboken, NJ 07030, USA.

Email address: mathxhli@hotmail.com, xiaohu.li@stevens.edu

** Postal address: School of Mathematical Sciences, Huaqiao University, Quanzhou, 362021, China. 
Suppose that a financial organization with the total capital $\hbar$ has a portfolio of several lines of business with potential losses $X_{1}, \ldots, X_{n}$. It is of great interest to allocate the capital $h_{i}$ to $X_{i}$ ( $i=1, \ldots, n$ and $\left.\hbar=h_{1}+\cdots+h_{n}>0\right)$ in a better manner so that the total potential loss may be minimized. To achieve this, researchers have proposed a number of ways to allocate the total capital, for example, the haircut allocation principle, the quantile allocation principle, the covariance allocation principle, the conditional tail expectation (CTE) allocation principle, and the proportional allocation principle. It should be noted here that only the covariance allocation principle, the CTE allocation principle, and the proportional allocation principle take the interdependence among multiple risks into consideration. For more details, we refer the reader to, e.g. Myers and Read (2001), Laeven and Goovaerts (2004), Frostig et al. (2007), Furman and Zitikis (2008), Tsanakas (2009), and Dhaene et al. (2012). Determining the appropriate capital allocation generally boils down to the direct optimization of the expected value of a function of concerned risks and the parameter vector concerning the allocation principle; see, for example, Dhaene et al. (2012). On the other hand, one may also formulate it in terms of the loss function. For example, Xu and $\mathrm{Hu}$ (2012) considered the total loss

$$
g(\boldsymbol{X}, \boldsymbol{h})=\sum_{i=1}^{n} \phi\left(X_{i}-h_{i}\right) \quad \text { for } \boldsymbol{h}=\left(h_{1}, \ldots, h_{n}\right) \text { with } h_{1}+\cdots+h_{n}=\hbar,
$$

where the convex function $\phi$ denotes the loss resulting from the deviation of loss $X_{i}$ from the allocated capital $h_{i}$. Under the framework of utility, the optimal capital allocation should minimize the expected utility of the total loss.

In the insurance industry, through paying a premium, the insured could obtain coverage from an insurance company. Two common forms of coverage are (ordinary) deductible and policy limit. One single insurance policy is usually designed to cover several random risks, say $X_{1}, \ldots, X_{n}$. In some situations, the policyholder has the right to allocate the deductibles or the policy limits among the involved risks. For example, as one of the main components of the compensation package of many insurance companies, the so-called 'flexible spending account programme' allows employees to allocate pre-tax dollars toward specific expenses such as health care, medical costs, or dependent care. In practice, as one inspiring plan, the insurer always grants the policyholder a total policy limit $\ell$, which can be allocated arbitrarily by the policyholder (say, $l_{i}$ to $X_{i}, i=1, \ldots, n$ ) across all the involved risks. In this setup, the policyholder incurs the total attained loss

$$
g(\boldsymbol{X}, \boldsymbol{l})=\sum_{i=1}^{n}\left(X_{i}-l_{i}\right)_{+} \quad \text { for } \boldsymbol{l}=\left(l_{1}, \ldots, l_{n}\right) \text { with } \sum_{i=1}^{n} l_{i}=\ell \text { and } x_{+}=\max \{x, 0\} .
$$

On the other hand, the policyholder is sometimes also provided with a total deductible amount $d$, and he/she may allocate a deductible amount $d_{i}$ to the risk $X_{i}, i=1, \ldots, n$, according to his/her own will. In this scenario, the policyholder incurs the total attained loss

$$
g(\boldsymbol{X}, \boldsymbol{d})=\sum_{i=1}^{n}\left(X_{i} \wedge d_{i}\right) \quad \text { for } \boldsymbol{d}=\left(d_{1}, \ldots, d_{n}\right) \text { with } \sum_{i=1}^{n} d_{i}=d \text { and } x \wedge y=\min \{x, y\} .
$$

Obviously, a policyholder prefers to choose an allocation policy with either a deductible or upper limit that minimizes the resulting total attained loss covered by the policy. There has been some research into the optimal allocation of policy limits and deductibles; see, for example, Cheung (2007), Hua and Cheung (2008b), Zhuang et al. (2009), Lu and Meng (2011), and the 
references therein. In general, these two problems can also be summarized as the optimization of the expected utility of the total attained loss incurred by the deductible or upper limit allocation.

In financial engineering, the theory of optimum portfolio allocation in response to multiple random risk assets is of practical interest and is rather extensive, at least where the expected utility framework is concerned. However, the implications of interdependence among random risk assets and distributional order in the assets allocation policy is still not well understood. Consider a risk investor with a portfolio of $n$ risk assets having potential returns $X_{1}, \ldots, X_{n}$, and assume that the investor allocates the amount $w_{i}$ of the entire wealth $\omega$ held by him/her to the risk asset $X_{i}, i=1, \ldots, n$. Then the investor attains the total potential return

$$
g(\boldsymbol{X}, \boldsymbol{w})=\sum_{i=1}^{n} w_{i} X_{i} \quad \text { for } \boldsymbol{w}=\left(w_{1}, \ldots, w_{n}\right) \text { with } \sum_{i=1}^{n} w_{i}=\omega .
$$

The optimal allocation of the portfolio is pursued in order to maximize the total potential return. In the framework of utility, this again gives rise to optimizing the expected utility of the total potential return. The portfolio allocation problem has been extensively discussed in the past twenty years. For further details of this line of research, we refer the reader to Landsberger and Meilijson (1990), Kijima and Ohnishi (1996), Hennessy and Lapan (2002), Cheung and Yang (2004), Cheung (2006), Chen and $\mathrm{Hu}$ (2008), Li and You (2014), and the references therein.

In the framework of utility theory, the above four types of optimal allocation issue can be summarized as the following optimization problem:

$$
\max _{\lambda_{1}+\cdots+\lambda_{n}=\lambda} \mathbb{E}[u(g(\boldsymbol{X}, \boldsymbol{\lambda}))] \quad \text { for any increasing utility } u \text {. }
$$

By substituting some specific real function $g(\boldsymbol{x}, \boldsymbol{\lambda})$ and a certain class of utility function $u$, we immediately obtain one of the above allocation problems, which is equivalent to the optimal parameter vector $\lambda$ (denoting the allocation policy), leading to the maximized function $g(\boldsymbol{X}, \boldsymbol{\lambda})$ in the sense of some specific stochastic orders. Without doubt, the general form (1.1) also includes some potential applications in other areas, such as redundance allocation in the theory of reliability and order allocation in inventory administration. We will focus on the portfolio allocation of capital, deductibles, the policy limit, and assets. Also, it is worth pointing out that, owing to the nonlinear nature of (1.1) or the partial aspect of the involved stochastic orders, the closed-form expression of the optimal allocation vector $\lambda$ is not available in a general context; however, some useful qualitative results concerning the relative size of each allocation may still be addressed to some extent.

The aim of this paper is twofold. On the one hand, we study the optimal allocation problem (1.1) with a more general function $g(\boldsymbol{x}, \lambda)$ and build some theoretical permutation theories on multiple random risks with interdependence. On the other hand, these theories are successfully applied to the optimal allocation problem in actuarial science, the insurance industry, and financial engineering. We obtain some insightful results, which either generalize or supplement the existing results in the literature and, hence, shed some light on the related line of research on risk management. It should be remarked here that these theories are of independent interest, as well as having excellent applications in financial engineering and actuarial science.

The rest of this paper is organized as follows. In Section 2 we review some basic concepts and several useful facts that we will use in developing our theoretical results. In Section 3 we present several permutation theorems concerning the expected utility of either the attained 
loss or potential profit corresponding to a certain allocation policy. In Section 4 we return to examine the capital allocation in actuarial science, the policy limit and deductible allocation in insurance, and portfolio allocation in financial engineering. In Section 4, we provide some insightful conclusions into the application of those theories that either enrich or improve the research in related fields.

Throughout this paper, the terms increasing and decreasing stand for nondecreasing and nonincreasing, respectively. In what follows, we use boldface uppercase and boldface lowercase letters to denote random vectors (e.g. $\boldsymbol{X}$ ) and real vectors (e.g. $\boldsymbol{x}$ ), respectively. Their elements, random variables on a probability space $(\Omega, \mathcal{F}, \mathrm{P})$ and real variables on $\mathbb{R}$, are represented by uppercase and lowercase letters (e.g. $X_{i}$ and $x_{i}$ ), respectively. Also, we denote by $\mathbf{1}(A)$ the indicator function on a set $A$, which takes the value 1 or 0 according to whether $A$ occurs or not, and $x \vee y=\max \{x, y\}$ and $x \wedge y=\min \{x, y\}$ will be used frequently in what follows. For convenience, we let $x_{+}=x \vee 0$, denote by $(x, \lambda)$ the vector concatenating the elements of $\boldsymbol{x}$ and $\boldsymbol{\lambda}$, and implicitly assumed that all expectations are finite whenever they appear.

\section{Preliminaries}

For ease of reference, in this section we recall some important notions such as stochastic orders, arrangement increasing property, and Archimedean copula, etc. The main theoretical results will be presented in terms of these concepts.

In economics and finance, stochastic orders play an important role in comparing inequalities and risks. For example, in economics the well-known utility theory may be stated in terms of the usual stochastic order and increasing concave order, and the increasing convex order serves as the main tool to compare two insurance risks. Without doubt, the magnitude of random risks is the utmost important factor in determining the optimal portfolio allocation in the sense of minimizing the potential loss in actuarial science or maximizing the prospective gain in financial engineering. In the following sections, we will show that stochastic orders are rather convenient and pertinent in making a judgement on the performance of the portfolio of either actuarial or financial risks.

A random variable $X$ with density $f$ is said to be smaller than a random variable $Y$ with density $g$ in the

(i) likelihood ratio order (denoted by $X \leq \operatorname{lr} Y$ ) if $g(x) / f(x)$ is increasing in $x$;

(ii) stochastic order (denoted by $X \leq_{\mathrm{st}} Y$ ) if $\mathbb{E}[\phi(X)] \leq \mathbb{E}[\phi(Y)]$ for any increasing $\phi$;

(iii) hazard rate order (denoted by $X \leq_{\mathrm{hr}} Y$ ) if $\bar{G}(x) / \bar{F}(x)$ is increasing in $x$;

(iv) reversed hazard rate order (denoted by $X \leq_{\mathrm{rh}} Y$ ) if $G(x) / F(x)$ is increasing in $x$;

(v) increasing concave (convex) order (denoted by $\left.X \leq_{\mathrm{icv}}\left(\leq_{\mathrm{icx}}\right) Y\right)$ if $\mathbb{E}[\phi(X)] \leq \mathbb{E}[\phi(Y)]$ for any increasing and concave (convex) $\phi$.

It should be remarked here that the likelihood ratio order, the reversed hazard rate order, the usual stochastic order, and the increasing concave order are referred to as the likelihood ratio dominance, the reversed hazard rate dominance, the first-order stochastic dominance, and the second-order stochastic dominance, respectively, in the economic and finance literature; see, for example, Kijima and Ohnishi (1996). Standard references for stochastic orders include Müller and Stoyan (2002), Denuit et al. (2005), and Shaked and Shanthikumar (2007). For recent advances of stochastic orders in reliability and risk, see $\mathrm{Li}$ and $\mathrm{Li}$ (2013). 
Both the capital allocation in actuarial science and portfolio allocation in financial engineering center on reasonably assigning certain amounts of capital to concerned risk losses or assets. In many such cases, permutation properties of the potential loss or gain have a key impact on the portfolio's performance.

For any permutation $\tau$ of $\{1, \ldots, n\}$ and a real vector $\boldsymbol{x}=\left(x_{1}, \ldots, x_{n}\right) \in \mathbb{R}^{n}$, let $\tau(\boldsymbol{x})=$ $\left(x_{\tau(1)}, \ldots, x_{\tau(n)}\right)$ be the permutated vector. Denote by $\boldsymbol{x}_{\uparrow}=\left(x_{[1]}, \ldots, x_{[n]}\right)$ and $\boldsymbol{x}_{\downarrow}=$ $\left(x_{[n]}, \ldots, x_{[1]}\right)$ the rearrangement vectors with the components of $\boldsymbol{x}$ being arranged in ascending and descending order, respectively.

A vector $(\boldsymbol{x}, \boldsymbol{\lambda})$ is said to be

(a) arrangement equivalent to the other vector $(\boldsymbol{y}, \boldsymbol{\gamma})$ (written as $(\boldsymbol{x}, \boldsymbol{\lambda}) \stackrel{\mathrm{a}}{=}(\boldsymbol{y}, \boldsymbol{\gamma})$ ) if there exists some permutation $\tau$ such that $(\tau(\boldsymbol{x}), \tau(\boldsymbol{\lambda}))=(\boldsymbol{y}, \boldsymbol{\gamma})$;

(b) smaller in the sense of the arrangement order than the other vector $(\boldsymbol{y}, \boldsymbol{\gamma})$ (written as $\left.(\boldsymbol{x}, \boldsymbol{\lambda}) \leq_{\mathrm{a}}(\boldsymbol{y}, \boldsymbol{\gamma})\right)$ if there are vectors $z_{i}=\left(z_{i, 1}, \ldots, z_{i, n}\right), i=1, \ldots, m$, such that (i) $(\boldsymbol{x}, \lambda) \stackrel{\mathrm{a}}{=}\left(\boldsymbol{x}_{\uparrow}, \boldsymbol{z}_{1}\right)$ and $(\boldsymbol{y}, \boldsymbol{\gamma}) \stackrel{\mathrm{a}}{=}\left(\boldsymbol{x}_{\uparrow}, \boldsymbol{z}_{m}\right)$ hold, and (ii) for each $i=1, \ldots, m$, there exists a pair $\{j, k\}$ with $1 \leq j<k \leq n$ such that $z_{i, k}=z_{i-1, j}>z_{i-1, k}=z_{i, j}$ and $z_{i, l}=z_{i-1, l}$ for $l \notin\{j, k\}$.

The arrangement order may be illustrated by the example

$$
\begin{aligned}
((7,5,3,1),(2,4,6,8)) & \stackrel{\mathrm{a}}{=}((1,3,5,7),(8,6,4,2)) \\
& \leq \mathrm{a}((1,3,5,7),(2,6,4,8)) \\
& \leq \mathrm{a}((1,3,5,7),(2,4,6,8)) .
\end{aligned}
$$

A real function $g(\boldsymbol{x}, \lambda): \mathbb{R}^{n} \times \mathbb{R}^{n} \mapsto \mathbb{R}$ is said to be arrangement increasing (AI) on $(\boldsymbol{x}, \boldsymbol{\lambda})$ if $g(\boldsymbol{x}, \boldsymbol{\lambda}) \leq g(\boldsymbol{y}, \boldsymbol{\gamma})$ whenever $(\boldsymbol{x}, \boldsymbol{\lambda}) \leq_{\mathrm{a}}(\boldsymbol{y}, \boldsymbol{\gamma}) ; g(\boldsymbol{x}, \boldsymbol{\lambda})$ is said to be arrangement decreasing (AD) when the above inequality is reversed.

The AI property on $(\boldsymbol{x}, \lambda)$ actually emphasizes the monotone property owing to the similarity on the arrangement of $\boldsymbol{x}$ and $\lambda$. According to Hollander et al. (1977),

$$
g_{1}(\boldsymbol{x}, \boldsymbol{\lambda})=\sum_{i=1}^{n} \lambda_{i} x_{i} \quad \text { and } \quad g_{2}(\boldsymbol{x}, \boldsymbol{\lambda})=\sum_{i=1}^{n}\left(x_{i} \wedge \lambda_{i}\right)
$$

are $\mathrm{AI}$ on $(\boldsymbol{x}, \lambda)$, and $g_{3}(\boldsymbol{x}, \lambda)=\sum_{i=1}^{n}\left(x_{i}-\lambda_{i}\right)_{+}$is $\mathrm{AD}$ on $(\boldsymbol{x}, \lambda)$. For more on the general theory of AI functions and their applications, see, e.g. Hollander et al. (1977), Boland and Proschan (1988), Boland et al. (1988), and Marshall et al. (2011).

When elements of $\lambda$ are in the ascending order, the AI property on $(\boldsymbol{x}, \boldsymbol{\lambda})$ is just the monotone property due to the similarity on the arrangement of $\boldsymbol{x}$ and the vector $(1, \ldots, n)$. Let

$$
\tau_{i j}\left(x_{1}, \ldots, x_{i}, \ldots, x_{j}, \ldots, x_{n}\right)=\left(x_{1}, \ldots, x_{j}, \ldots, x_{i}, \ldots, x_{n}\right)
$$

for $1 \leq i<j \leq n$. Let us introduce the permutation monotone property of a multivariate function.

Definition 2.1. A function $g(\boldsymbol{x}): \mathbb{R}^{n} \mapsto \mathbb{R}$ is said to be permutation decreasing (PD) with respect to $(i, j)$ with $i<j$ if $g(\boldsymbol{x}) \geq g\left(\tau_{i j}(\boldsymbol{x})\right)$ for $x_{i} \leq x_{j} ; g$ is said to be permutation increasing (PI) with respect to $(i, j)$ with $i<j$ when the inequality is reversed. 
The PD (PI) property actually asserts that, for $(i, j)$, the function attains a greater (smaller) value when $\left(x_{i}, x_{j}\right)$ and $(i, j)$ are similarly ordered with other arguments unchanged. Recall that a function $g(\boldsymbol{x}): \mathbb{R}^{n} \mapsto \mathbb{R}$ is said to be AI in $\boldsymbol{x}$ if

$$
\left(x_{i}-x_{j}\right)\left[g(\boldsymbol{x})-g\left(\tau_{i j}(\boldsymbol{x})\right)\right] \leq 0 \text { for all } i \text { and } j \text { such that } 1 \leq i<j \leq n ;
$$

it is said to be AD in $\boldsymbol{x}$ when the above inequality is reversed.

Evidently, the AI (AD) property of a function $g(\boldsymbol{x})$ is just its PD (PI) properties with respect to any pair $(i, j)$ with $1 \leq i<j \leq n$. For more on the $\mathrm{AI}$ and AD properties, see Boland et al. (1988) and Marshall et al. (2011). Here we introduce the following two weak versions of the PD and PI properties; they play a critical role in developing our main theoretical results.

Definition 2.2. A joint density $f(\boldsymbol{x})$ is said to be

(i) upper tail permutation decreasing (UTPD) with respect to $(i, j)$ with $i<j$ if

$$
\int_{t}^{\infty}\left[f(\boldsymbol{x})-f\left(\tau_{i j}(\boldsymbol{x})\right)\right] \mathrm{d} x_{j} \geq 0 \quad \text { for all } t \geq x_{i} \text { and any fixed } x_{i}
$$

(ii) lower tail permutation decreasing (LTPD) with respect to $(i, j)$ with $i<j$ if

$$
\int_{-\infty}^{t}\left[f(\boldsymbol{x})-f\left(\tau_{i j}(\boldsymbol{x})\right)\right] \mathrm{d} x_{i} \geq 0 \quad \text { for all } t \geq x_{i} \text { and any fixed } x_{i} .
$$

The joint density $f(\boldsymbol{x})$ is said to be upper tail permutation increasing (UTPI) and lower tail permutation increasing (LTPI) with respect to $i<j$ if the above two inequalities are reversed.

Note that, for some given $(i, j)$ with $i<j$, the PD (PI) property always implies the UTPD and LTPD (UTPI and LTPI) properties.

Another factor that has a vital impact on the performance of the portfolio in economics, finance, and insurance is the interdependence among concerned multiple random risks. Classical models usually assume the mutual independence among them; however, it is obviously inappropriate in practice. In the last two decades, the copula theory has gained more and more attention in areas dealing with risks due to its simple mathematical form and statistical tractability.

Denote by $\boldsymbol{X}=\left(X_{1}, \ldots, X_{n}\right)$ a random vector with marginal distributions $F_{1}, \ldots, F_{n}$. If there exists a joint distribution function $C$ on the $n$-dimensional unit square $[0,1]^{n}$ such that the joint distribution of $\boldsymbol{X}$ may be represented as $F\left(x_{1}, \ldots, x_{n}\right)=C\left(F_{1}\left(x_{1}\right), \ldots, F_{n}\left(x_{n}\right)\right)$ for any $x_{1}, \ldots, x_{n}$, then $C$ is called the copula of $\boldsymbol{X}$. If there exists some joint distribution function $\widehat{C}$ on the $n$-dimensional unit square $[0,1]^{n}$ such that the survival function $\bar{F}$ of $\boldsymbol{X}$ may be represented as $\bar{F}(\boldsymbol{x})=\widehat{C}\left(\bar{F}_{1}\left(x_{1}\right), \ldots, \bar{F}_{n}\left(x_{n}\right)\right)$ for all $x_{i}, i=1, \ldots, n$, then $\widehat{C}$ is called the survival copula of $\boldsymbol{X}$.

Recently, much attention has been paid to Archimedean copulas due to the mathematical tractability and flexibility in specifying the dependence structure of multivariate distributions. As one of the most important family of copulas, the Archimedean copula was first introduced by Kimberling (1974), and studied at closer range by Genest and MacKay (1986a), (1986b), Marshall and Olkin (1988), and Genest and Rivest (1993), among others.

A function $\varphi$ defined on $(0,+\infty)$ is said to be $n$-monotone if $(-1)^{k} \varphi^{(k)}(t) \geq 0$ for $t \in$ $(0,+\infty)$, where $\varphi^{(0)}(t)=\varphi(t)$ and $\varphi^{(k)}(t)$ is the $k$ th-order derivative evaluated at $t, k=$ $1, \ldots, n$. Obviously, for an $n$-monotone function $\varphi(t),(-1)^{k} \varphi^{(k)}(t)$ is always decreasing in $t$ for any $k=1, \ldots, n-1$. 
Definition 2.3. (McNeil and Nešlehová (2009).) For an $n$-monotone function $\varphi$ : $[0,+\infty)$ $\mapsto(0,1]$ with $\varphi(0)=1$ and $\lim _{t \rightarrow \infty} \varphi(t)=0$, the function $C\left(u_{1}, \ldots, u_{n}\right)=\varphi\left(\varphi^{-1}\left(u_{1}\right)\right.$ $\left.+\cdots+\varphi^{-1}\left(u_{n}\right)\right)$ is called the Archimedean copula with generator $\varphi$.

Assume that $X_{i}$ has the distribution function $F_{i}$ and the density function $f_{i}, i=1, \ldots, n$. Let $\psi=\varphi^{-1}:(0,1] \rightarrow[0,+\infty)$, and, for $i=1, \ldots, n$, let

$$
s_{i}(x)=\psi^{(1)}\left(F_{i}(x)\right) f_{i}(x) \quad \text { and } \quad s_{i}^{*}(x)=\psi^{(1)}\left(\bar{F}_{i}(x)\right) f_{i}(x) .
$$

Then $\boldsymbol{X}$ with the Archimedean copula generated by $\varphi$ has the joint distribution $F(\boldsymbol{x})=$ $\varphi\left(\psi\left(F_{1}\left(x_{1}\right)\right)+\cdots+\psi\left(F_{n}\left(x_{n}\right)\right)\right)$ and the probability density

$$
f(\boldsymbol{x})=\varphi^{(n)}\left(\sum_{i=1}^{n} \psi\left(F_{i}\left(x_{i}\right)\right)\right) \prod_{i=1}^{n} s_{i}\left(x_{i}\right),
$$

and $\boldsymbol{X}$ with the Archimedean survival copula generated by $\varphi$ has the survival function $\bar{F}(\boldsymbol{x})=$ $\varphi\left(\psi\left(\bar{F}_{1}\left(x_{1}\right)\right)+\cdots+\psi\left(\bar{F}_{n}\left(x_{n}\right)\right)\right)$ and the probability density

$$
f(\boldsymbol{x})=\varphi^{(n)}\left(\sum_{i=1}^{n} \psi\left(\bar{F}_{i}\left(x_{i}\right)\right)\right) \prod_{i=1}^{n} s_{i}^{*}\left(x_{i}\right) .
$$

In the remainder of this paper, we will handle Archimedean copulas with a log-convex generator. It is easily verified that the log-convex generator of bivariate Archimedean survival copulas leads to a right-tail increasing property and that the log-convex generator of bivariate Archimedean copulas leads to a left-tail decreasing property-two positive dependence relations due to Esary and Proschan (1972). The theory of copulas is quite useful in modeling the dependence of multivariate distributions, and it is the standard tool in handling interdependence in statistics. See Joe (1997), McNeil et al. (2005), and Nelsen (2006) for comprehensive studies on copula theory and its applications.

\section{Theoretical results}

Since the optimization problems introduced in Section 1 share a unified mathematical form (1.1), the expectation of a real function of the risk vector, and the parameter vector of allocation in the portfolio, we provide new insight to some aspects of the optimality of the portfolio allocation by pursuing the essence of the pure mathematical issue.

The first theory due to Boland et al. (1988) presents the stochastic order of the AI function of a random vector with AI joint density. As will be seen, we play a new tune on this ancient flute in the next section.

Theorem 3.1. (Boland et al. (1988, Corollary 3.1).) Suppose that a real function $g(\boldsymbol{x}, \lambda)$ is $A I$ on $(\boldsymbol{x}, \lambda)$ and that a random vector $\boldsymbol{X}=\left(X_{1}, \ldots, X_{n}\right)$ has an AI joint density $f(\boldsymbol{x})$. Then it holds that $g(\boldsymbol{X}, \boldsymbol{\lambda}) \geq_{\mathrm{st}} g\left(\boldsymbol{X}, \tau_{i j}(\boldsymbol{\lambda})\right)$ whenever $\lambda_{i} \leq \lambda_{j}$ for $1 \leq i<j \leq n$.

For risk-neutral investors with AI total risk return, Theorem 3.1 proposes a better portfolio or capital allocation for multiple risks with an AI joint density. Now, let us proceed to two main theoretical results on multiple risks having the UTPD (UTPI) or LTPD (LTPI) joint density, which will be applied to study the optimal portfolio allocations in the next section. The first result involves finding sufficient conditions on the increasing convex order of the function of a random vector $\boldsymbol{X}$ that defines the multiple risks, and a real parameter vector $\lambda$ of the allocation policy. 
Theorem 3.2. Suppose that $g(\boldsymbol{x}, \boldsymbol{\lambda}): \mathbb{R}^{n} \times \mathbb{R}^{n} \rightarrow \mathbb{R}$ is $A I(A D)$ on $(\boldsymbol{x}, \boldsymbol{\lambda})$ and that the joint density of a random vector $\boldsymbol{X}$ has the UTPD (UTPI) property with respect to a pair $(i, j)$ with $i>j(i<j)$. For the pair $(i, j)$, if both $g(\boldsymbol{x}, \lambda)$ and $g(\boldsymbol{x}, \lambda)-g\left(\boldsymbol{x}, \tau_{i j}(\boldsymbol{\lambda})\right)$ are increasing in $x_{i} \geq x_{j}$ and $\lambda_{i} \geq(\leq) \lambda_{j}$, then $g(X, \lambda) \geq_{\text {icx }}\left(\leq_{\text {icx }}\right) g\left(X, \tau_{i j}(\lambda)\right)$ whenever $\lambda_{i} \geq \lambda_{j}$.

The above theorem provides risk-seeking investors that have an AI total risk return with a guide to allocating better their capital in a portfolio of multiple risks. It should be pointed out here that, for a pair $(i, j)$ with $i>j$, the UTPD property of $\boldsymbol{X}$ is invoked when either of the following two assumptions holds.

(i) $\boldsymbol{X}$ has an Archimedean survival copula with $s_{i}^{*}(x) \geq s_{j}^{*}(x)$ for $1 \leq j<i \leq n$.

(ii) All $(n-1)$-dimensional marginal distributions of $\boldsymbol{X}$ are identical and

$$
\left(X_{i} \mid \boldsymbol{X}_{(i)}=\boldsymbol{x}_{(i)}\right) \geq_{\mathrm{st}}\left(X_{j} \mid \tau_{i j}(\boldsymbol{X})_{(i)}=\boldsymbol{x}_{(i)}\right) \text { for } 1 \leq j<i \leq n,
$$

where $\boldsymbol{x}_{(i)}=\left(x_{1}, \ldots, x_{i-1}, x_{i+1}, \ldots, x_{n}\right)$.

Assumption (i) follows directly from the proof of Corollary 3.1. As for assumption (ii), note that (3.1) is equivalent to

$$
f_{\tau_{i j}(\boldsymbol{X})_{(i)}}\left(\boldsymbol{x}_{(i)}\right) \int_{t}^{\infty} f(\boldsymbol{x}) \mathrm{d} x_{i} \geq f_{\boldsymbol{X}_{(i)}}\left(\boldsymbol{x}_{(i)}\right) \int_{t}^{\infty} f\left(\tau_{i j}(\boldsymbol{x})\right) \mathrm{d} x_{i} \quad \text { for } t \geq 0,
$$

and all $(n-1)$-dimensional marginal densities of $\boldsymbol{X}$ are identical. Then we immediately have $f_{\tau_{i j}(\boldsymbol{X})_{(i)}}\left(\boldsymbol{x}_{(i)}\right)=f_{\boldsymbol{X}_{(i)}}\left(\boldsymbol{x}_{(i)}\right)$ and, hence, the joint density has the UTPD property on the pair $(i, j)$.

From Theorem 3.2, we develop the following corollary concerning the multiple random variables with an Archimedean survival copula.

Corollary 3.1. Suppose that $\boldsymbol{X}$ has an Archimedean survival copula and that $g(\boldsymbol{x}, \boldsymbol{\lambda})$ satisfies the conditions of Theorem 3.2. Then $s_{i}^{*}(x) \geq s_{j}^{*}(x)$ for all $x \geq 0$ and $1 \leq i, j \leq n$, implying that $g(\boldsymbol{X}, \lambda) \geq_{\mathrm{icx}}\left(\leq_{\mathrm{icx}}\right) g\left(\boldsymbol{X}, \tau_{i j}(\lambda)\right)$ whenever $\lambda_{i} \geq \lambda_{j}$.

As an immediate consequence of Corollary 3.1, we obtain the following corollary concerning multiple random variables arrayed according to the hazard rate order and equipped with an Archimedean survival copula.

Corollary 3.2. Assume that $\boldsymbol{X}$ has an Archimedean survival copula with a log-convex generator and that $g(\boldsymbol{x}, \lambda)$ satisfies the related conditions of Theorem 3.2. Then for $1 \leq i, j \leq n$, $X_{i} \geq_{\mathrm{hr}} X_{j}$ implies that $g(\boldsymbol{X}, \boldsymbol{\lambda}) \geq_{\mathrm{icx}}\left(\leq_{\mathrm{icx}}\right) g\left(\boldsymbol{X}, \tau_{i j}(\boldsymbol{\lambda})\right)$ whenever $\lambda_{i} \geq \lambda_{j}$.

Now we proceed to the second main theoretical result.

Theorem 3.3. Suppose that a real function $g(\boldsymbol{x}, \lambda): \mathbb{R}^{n} \times \mathbb{R}^{n} \mapsto \mathbb{R}$ is $A I(A D)$ on $(\boldsymbol{x}, \boldsymbol{\lambda})$ and that the joint density of a random vector $X$ is LTPD or LTPI with respect to a pair $(i, j)$ with $i>j$ or $i<j$. For the pair $(i, j)$, if $g(\boldsymbol{x}, \lambda)$ and $g\left(\boldsymbol{x}, \tau_{i j}(\boldsymbol{\lambda})\right)-g(\boldsymbol{x}, \boldsymbol{\lambda})$ are increasing in $x_{j} \leq x_{i}$ and $\lambda_{i} \geq(\leq) \lambda_{j}$, then $g(\boldsymbol{X}, \lambda) \geq_{\text {icv }}\left(\leq_{\text {icv }}\right) g\left(X, \tau_{i j}(\lambda)\right)$ whenever $\lambda_{i} \geq \lambda_{j}$.

In parallel to Theorem 3.2, Theorem 3.3 provides risk-averse investors that have an AI total risk return with a guide to better allocating their capital in a portfolio of multiple risks. Likewise, it is easy to verify that, for a pair $(i, j)$ with $i>j$, the LTPD property of $\boldsymbol{X}$ is followed when either of the next two statements holds. 
(i) $\boldsymbol{X}$ has an Archimedean copula and $s_{i}(x) \leq s_{j}(x)$ for $1 \leq j<i \leq n$.

(ii) All ( $n-1)$-dimensional marginals of $\boldsymbol{X}$ have identical distributions and (3.1) is satisfied.

The remaining results concerning random vectors with an Archimedean copula follow as a consequence of Theorem 3.3.

Corollary 3.3. Assume that $\boldsymbol{X}$ has an Archimedean copula. If $g(\boldsymbol{x}, \boldsymbol{\lambda})$ satisfies the related conditions in Theorem 3.3, then $s_{i}(x) \leq s_{j}(x)$ for all $x \geq 0$ and $1 \leq i, j \leq n$, implying that $g(\boldsymbol{X}, \boldsymbol{\lambda}) \geq_{\mathrm{icv}}\left(\leq_{\mathrm{icv}}\right) g\left(\boldsymbol{X}, \tau_{i j}(\boldsymbol{\lambda})\right)$ whenever $\lambda_{i} \geq \lambda_{j}$.

Moreover, we present a result based on the reversed hazard rate order.

Corollary 3.4. Assume that $\boldsymbol{X}$ has an Archimedean copula with a log-convex generator. If $g(\boldsymbol{x}, \lambda)$ satisfies the related conditions in Theorem 3.3, then for $1 \leq i, j \leq n, X_{i} \geq_{\mathrm{rh}} X_{j}$ implies that $g(\boldsymbol{X}, \boldsymbol{\lambda}) \geq_{\mathrm{icv}}\left(\leq_{\mathrm{icv}}\right) g\left(\boldsymbol{X}, \tau_{i j}(\boldsymbol{\lambda})\right)$ whenever $\lambda_{i} \geq \lambda_{j}$.

\section{Applications in actuarial and financial risk management}

In this section we apply the theoretical results presented in Section 3 to capital allocation in actuarial science, the deductible and upper limit allocation in the insurance industry, and the portfolio allocation in financial engineering. For convenience, throughout this section, all random variables $X_{i}$ are assumed to be nonnegative, and we denote, for any $\lambda>0$, the simplex

$$
\mathcal{A}_{\lambda}=\left\{\left(\lambda_{1}, \ldots, \lambda_{n}\right): \lambda_{1}+\cdots+\lambda_{n}=\lambda, \lambda_{i} \geq 0, i=1, \ldots, n\right\}
$$

as the set of all feasible allocation policies.

\subsection{Actuarial risk capital allocation}

In the literature on actuarial science, the optimal capital allocation has been extensively discussed. Based on the marginal cost and tail value-at-risk, Panjer (2001) was the first to provide an explicit expression for allocations of multivariate normally distributed risks, and Landsman and Valdez (2003) extended this result to multivariate elliptical distributions. Tsanakas (2004) subsequently studied capital allocations based on the distorted risk measure, and Tsanakas (2009) further generalized these allocation principles to the general convex risk measures. Moreover, Dhaene et al. (2012) developed a unified framework for capital allocations.

Here we focus on the following model:

$$
\min _{\boldsymbol{h} \in \mathcal{A}_{\hbar}} \mathbb{E}\left[u\left(\sum_{i=1}^{n} \phi\left(X_{i}-h_{i}\right)\right)\right] \text { for any increasing } u \text {, convex } \phi \text {, and } \hbar>0 .
$$

Let $\boldsymbol{h}^{*}=\left(h_{1}^{*}, \ldots, h_{n}^{*}\right)$ be an optimal solution to problem (4.1). Recently, Xu and Hu (2012) provided the following result.

Proposition 4.1. ( $\mathrm{Xu}$ and $\mathrm{Hu}\left(2012\right.$, Theorem 4.6).) Suppose that $X_{1}, \ldots, X_{n}$ are mutually independent. Then $X_{i} \leq \operatorname{lr} X_{j}$ implies that $h_{i}^{*} \leq h_{j}^{*}$ for any $1 \leq i, j \leq n$.

From Proposition 4.1, if $X_{1}, \ldots, X_{n}$ are mutually independent and $X_{1} \leq_{1 \mathrm{r}} \cdots \leq_{\mathrm{lr}} X_{n}$, then the optimal capital allocation policy $\boldsymbol{h}^{*}$ for (4.1) should satisfy $h_{i}^{*} \leq h_{j}^{*}$ for any $1 \leq i<j \leq n$. According to Hua and Cheung $(2008 \mathrm{a}),\left(X_{1}, \ldots, X_{n}\right)$ has an AI joint density if they are mutually independent and $X_{1} \leq_{\mathrm{lr}} \cdots \leq_{\mathrm{lr}} X_{n}$; Proposition 4.2 below provides a more general conclusion on the optimal allocation policy for (4.1). 
Proposition 4.2. Suppose that $\left(X_{1}, \ldots, X_{n}\right)$ has an AI joint density. Then the optimal capital allocation vector $\boldsymbol{h}^{*}$ satisfies $h_{i}^{*} \leq h_{j}^{*}$ for any $1 \leq i<j \leq n$.

Proof. Let $g(x, h)=-\phi(x-h)$. For $x_{i} \geq x_{j}$ and $h_{i} \geq h_{j}$, it holds that

$$
\begin{aligned}
& x_{i} \vee x_{j}-h_{i} \wedge h_{j} \geq x_{i} \vee x_{j}-h_{i} \vee h_{j}, \\
& x_{i} \wedge x_{j}-h_{i} \wedge h_{j} \geq x_{i} \wedge x_{j}-h_{i} \vee h_{j}, \\
& x_{i} \vee x_{j}-h_{i} \wedge h_{j} \geq x_{i} \wedge x_{j}-h_{i} \wedge h_{j}
\end{aligned}
$$

By the convexity of $\phi$, we have

$$
\begin{aligned}
& \phi\left(x_{i} \vee x_{j}-h_{i} \wedge h_{j}\right)-\phi\left(x_{i} \vee x_{j}-h_{i} \vee h_{j}\right) \\
& \quad \geq \phi\left(x_{i} \wedge x_{j}-h_{i} \wedge h_{j}\right)-\phi\left(x_{i} \wedge x_{j}-h_{i} \vee h_{j}\right),
\end{aligned}
$$

which is equivalent to

$$
\begin{aligned}
& g\left(x_{i} \vee x_{j}, h_{i} \vee h_{j}\right)+g\left(x_{i} \wedge x_{j}, h_{i} \wedge h_{j}\right) \\
& \quad \geq g\left(x_{i} \vee x_{j}, h_{i} \wedge h_{j}\right)+g\left(x_{i} \wedge x_{j}, h_{i} \vee h_{j}\right) .
\end{aligned}
$$

Then from Proposition 2.5(b) of Boland et al. (1988) and (4.2), it follows that

$$
\sum_{i=1}^{n} g\left(x_{i}, h_{i}\right)=-\sum_{i=1}^{n} \phi\left(x_{i}-h_{i}\right)
$$

is $\mathrm{AI}$ on $(\boldsymbol{x}, \boldsymbol{h})$. As a result of Theorem 3.1, it follows that, for $1 \leq i<j \leq n$ and $h_{i} \leq h_{j}$,

$$
-\sum_{k=1}^{n} \phi\left(X_{k}-h_{k}\right) \geq_{\mathrm{st}}-\phi\left(X_{i}-h_{j}\right)-\phi\left(X_{j}-h_{i}\right)-\sum_{k \notin\{i, j\}}^{n} \phi\left(X_{k}-h_{k}\right) .
$$

This is just

$$
\sum_{k=1}^{n} \phi\left(X_{k}-h_{k}\right) \leq_{\mathrm{st}} \phi\left(X_{i}-h_{j}\right)+\phi\left(X_{j}-h_{i}\right)+\sum_{k \notin\{i, j\}}^{n} \phi\left(X_{k}-h_{k}\right)
$$

for $i<j$ and $h_{i} \leq h_{j}$. So, we obtain the desired result.

In parallel, we also present an application of Corollary 3.2.

Proposition 4.3. Assume that $u$ is convex and that $\boldsymbol{X}$ has an Archimedean survival copula with a log-convex generator. If $\phi$ is increasing and convex, then $X_{i} \geq_{\mathrm{hr}} X_{j}$ implies that $h_{i}^{*} \geq h_{j}^{*}$ for $1 \leq i, j \leq n$.

Proof. The increasing property of $\phi$ guarantees that $g(\boldsymbol{x}, \boldsymbol{h}):=\sum_{i=1}^{n} \phi\left(x_{i}-h_{i}\right)$ increases in $x_{i}$. From the proof of Proposition 4.2, it follows that $g(\boldsymbol{x}, \boldsymbol{h})$ is AD on $(\boldsymbol{x}, \boldsymbol{h})$. Since $\phi$ is convex, $\phi\left(x_{i}-h_{i}\right)-\phi\left(x_{i}-h_{j}\right)$ is increasing in $x_{i}$ for $h_{i} \leq h_{j}$, and, hence,

$$
g(\boldsymbol{x}, \boldsymbol{h})-g\left(\boldsymbol{x}, \tau_{i j}(\boldsymbol{h})\right)=\phi\left(x_{i}-h_{i}\right)-\phi\left(x_{i}-h_{j}\right)+\phi\left(x_{j}-h_{j}\right)-\phi\left(x_{j}-h_{i}\right)
$$


increases in $x_{i}$ for $h_{i} \leq h_{j}$. That is, $g(\boldsymbol{x}, \boldsymbol{h})$ satisfies the related conditions of Theorem 3.2 for $h_{i} \leq h_{j}$. Consequently, from Corollary 3.2, $X_{i} \geq_{\mathrm{hr}} X_{j}$ implies that, for $h_{i} \geq h_{j}$ and $1 \leq i, j \leq n$,

$$
\sum_{k=1}^{n} \phi\left(X_{k}-h_{k}\right) \leq_{\mathrm{icx}} \phi\left(X_{i}-h_{j}\right)+\phi\left(X_{j}-h_{i}\right)+\sum_{k \notin\{i, j\}}^{n} \phi\left(X_{k}-h_{k}\right) .
$$

Thus, it follows that $h_{i}^{*} \geq h_{j}^{*}$ for $1 \leq i, j \leq n$.

\subsection{Insurance upper limits and deductibles allocation}

For the allocation of upper limits and deductibles in the insurance industry, the optimal problem (1.1) can be represented as

$$
\min _{l \in \mathcal{A}_{\ell}} \mathbb{E}\left[u\left(\sum_{i=1}^{n}\left(X_{i}-l_{i}\right)_{+}\right)\right] \text {for any increasing } u \text { and } \ell>0
$$

and

$$
\min _{\boldsymbol{d} \in \mathcal{A}_{d}} \mathbb{E}\left[u\left(\sum_{i=1}^{n}\left(X_{i} \wedge d_{i}\right)\right)\right] \text { for any increasing } u \text { and } d>0 .
$$

Let $\boldsymbol{l}^{*}=\left(l_{1}^{*}, \ldots, l_{n}^{*}\right)$ and $\boldsymbol{d}^{*}=\left(d_{1}^{*}, \ldots, d_{n}^{*}\right)$ be the optimal solutions to (4.3) and (4.4), respectively. Cheung (2007), who was one of the first to study the optimal allocation of policy limits and deductibles, provided the following result on mutually independent random risks.

Proposition 4.4. (Cheung (2007, Propositions 1 and 4).) Assume that $u$ is convex and that $X_{1}, \ldots, X_{n}$ are mutually independent. Then $X_{i} \geq \mathrm{hr} X_{j}$ implies that $l_{i}^{*} \geq l_{j}^{*}$ and $d_{i}^{*} \leq d_{j}^{*}$ for $1 \leq i, j \leq n$.

From the viewpoint of the insurer, Hua and Cheung (2008b) investigated the worst allocation of policy limits and deductibles. Zhuang et al. (2009) then established some nice complements and extensions for the main results in Cheung (2007) and Hua and Cheung (2008b). The following most recent result is due to Lu and Meng (2011).

Proposition 4.5. (Lu and Meng (2011, Lemmas 5.1 and 5.3).) Assume that the random risks $X_{1}, \ldots, X_{n}$ are mutually independent. Then $X_{i} \leq_{\operatorname{lr}} X_{j}$ implies that $l_{i}^{*} \leq l_{j}^{*}$ and $d_{i}^{*} \geq d_{j}^{*}$ for $1 \leq i, j \leq n$.

Next, we establish a generalization of Proposition 4.5.

Proposition 4.6. Suppose that $\left(X_{1}, \ldots, X_{n}\right)$ has an AIjoint density. Then $l_{i}^{*} \leq l_{j}^{*}$ and $d_{i}^{*} \geq d_{j}^{*}$ for any $1 \leq i<j \leq n$.

Proof. Since $\phi(x)=x_{+}$is convex, by Proposition 4.2, we immediately obtain $l_{i}^{*} \leq l_{j}^{*}$ for any $1 \leq i<j \leq n$.

From (2.1) and Theorem 3.1, the AI property of the joint density implies that, for any $1 \leq i<j \leq n$ and $d_{i} \leq d_{j}$,

$$
\sum_{k=1}^{n}\left(X_{k} \wedge d_{k}\right) \geq_{\mathrm{st}}\left(X_{i} \wedge d_{j}\right)+\left(X_{j} \wedge d_{i}\right)+\sum_{k \notin\{i, j\}}\left(X_{k} \wedge d_{k}\right) .
$$

This results in $d_{i}^{*} \geq d_{j}^{*}$ for any $1 \leq i<j \leq n$ and, hence, completes the proof. 
The next result serves as an extension of Proposition 4.4 in the sense of relaxing the independence among $\boldsymbol{X}$ to the interdependence through an Archimedean copula.

Proposition 4.7. Assume that $u$ is convex and that $\boldsymbol{X}$ has an Archimedean survival copula with a log-convex generator. Then $X_{i} \geq{ }_{\mathrm{hr}} X_{j}$ implies that $l_{i}^{*} \geq l_{j}^{*}$ and $d_{i}^{*} \leq d_{j}^{*}$ for $1 \leq i, j \leq n$.

Proof. Let $g(\boldsymbol{x}, \boldsymbol{d})=\sum_{i=1}^{n}\left(x_{i} \wedge d_{i}\right)$, which is obviously increasing in $x_{i}$. From (2.1), $g(\boldsymbol{x}, \boldsymbol{d})$ is AI on $(\boldsymbol{x}, \boldsymbol{d})$. For $d_{i} \geq d_{j}$,

$$
\begin{aligned}
g(\boldsymbol{x}, \boldsymbol{d})-g\left(\boldsymbol{x}, \tau_{i j}(\boldsymbol{d})\right) & =x_{i} \wedge d_{i}+x_{j} \wedge d_{j}-x_{i} \wedge d_{j}-x_{j} \wedge d_{i} \\
& = \begin{cases}x_{j} \wedge d_{j}-x_{j} \wedge d_{i}, & x_{i} \leq d_{j}, \\
x_{i}-d_{j}+x_{j} \wedge d_{j}-x_{j} \wedge d_{i}, & d_{j} \leq x_{i} \leq d_{i}, \\
d_{i}-d_{j}+x_{j} \wedge d_{j}-x_{j} \wedge d_{i}, & d_{i} \leq x_{i},\end{cases}
\end{aligned}
$$

is increasing in $x_{i}$ and, hence, $g(\boldsymbol{x}, \boldsymbol{d})$ satisfies the conditions of Theorem 3.2 for $d_{i} \geq d_{j}$. By Corollary 3.2, $X_{i} \geq{ }_{\mathrm{hr}} X_{j}$ implies that, for $d_{i} \geq d_{j}$ and $1 \leq i, j \leq n$,

$$
\sum_{k=1}^{n}\left(X_{k} \wedge d_{k}\right) \geq_{\mathrm{icx}}\left(X_{i} \wedge d_{j}\right)+\left(X_{j} \wedge d_{i}\right)+\sum_{k \notin\{i, j\}}\left(X_{k} \wedge d_{k}\right)
$$

It then follows that $d_{i}^{*} \leq d_{j}^{*}$ for $1 \leq i, j \leq n$.

Note that $\phi(x)=x_{+}$is increasing and convex, and recall from Proposition 4.3 that $X_{i} \geq_{\mathrm{hr}} X_{j}$. Then $l_{i}^{*} \geq l_{j}^{*}$ for $1 \leq i, j \leq n$.

It should be remarked here that the independent copula has the log-convex generator $\varphi(x)=$ $\mathrm{e}^{-x}$, and, thus, Proposition 4.4 is substantially extended by Proposition 4.7.

\subsection{Financial portfolio allocation based on utility}

For financial portfolio allocations, the optimization problem (1.1) becomes

$$
\max _{\boldsymbol{w} \in \mathcal{A}_{\omega}} \mathbb{E}\left[u\left(\sum_{i=1}^{n} w_{i} X_{i}\right)\right] \text { for any increasing } u \text { and } \omega>0 .
$$

Let $\boldsymbol{w}^{*}=\left(w_{1}^{*}, \ldots, w_{n}^{*}\right)$ be an optimal solution to (4.5). Landsberger and Meilijson (1990) provided the following pioneering result.

Proposition 4.8. (Landsberger and Meilijson (1990, Proposition 1).) Suppose that the random risks $X_{1}, \ldots, X_{n}$ are mutually independent. Then $X_{i} \leq \operatorname{lr} X_{j}$ implies that $w_{i}^{*} \leq w_{j}^{*}$ for $1 \leq$ $i, j \leq n$.

In a similar manner, Kijima and Ohnishi (1996) proved that the shares of the portfolio allocation should be ordered whenever the corresponding returns are arrayed according to the reversed hazard rate order. Subsequently, Hennessy and Lapan (2002) were the first to study the risk-averter's portfolio allocation under the assumption of the Archimedean copula for potential returns of risk assets and developed the following result.

Proposition 4.9. (Lapan and Hennessy (2002, Proposition 4.2).) Suppose that $u$ is concave and twice continuously differentiable, and that the random risks $X_{1}, \ldots, X_{n}$ have an Archimedean copula with a log-convex generator. Then $X_{i} \geq_{\mathrm{rh}} X_{j}$ implies that $w_{i}^{*} \geq w_{j}^{*}$ for $1 \leq i, j \leq n$. 
In addition, Cheung and Yang (2004) discussed the assets modeled by dependent default risks by considering both the threshold model and the independent model of risks driven by default indicators. They provided the condition on the default indicators sufficient to the same order as in the optimal allocation. Moreover, instead of assuming any specific dependent structure, Cheung (2006) proposed the maximin criterion as an alternative optimization criterion, which first identifies the least favorable dependence structure as the actual one, and then proceeds to analyze the optimal portfolio allocation for this situation. In the context of dependent returns driven by default indicators, Chen and $\mathrm{Hu}$ (2008) established sufficient conditions on the dependence of indicators and the utility function for the optimal allocation vector to be ordered. Recently, Li and You (2014) investigated the risk-neutral's portfolio allocation under the assumption that the returns are coupled by some Archimedean copula.

Let $l_{x_{i}}$ and $u_{x_{i}}$ be the left and right endpoints of its support, $i=1, \ldots, n$. For convenience, let $l_{i j}=\max \left\{l_{x_{i}}, l_{x_{j}}\right\}$ and $u_{i j}=\min \left\{u_{x_{i}}, u_{x_{j}}\right\}$ for $1 \leq i<j \leq n$.

Proposition 4.10. ( $\mathrm{Li}$ and You (2014, Theorem 5).) Suppose that $\boldsymbol{X}$ has an Archimedean copula with $(n+1)$-monotone $\varphi$ such that $(i)(1-t) \psi^{(2)}(t) / \psi^{(1)}(t)$ is decreasing in $t$, and (ii) for $1 \leq i<j \leq n, s_{i}(t) / s_{j}(t) \rightarrow 1$ as $t \rightarrow l_{i j}$. Then $X_{i} \leq \operatorname{lr} X_{j}$ implies that $w_{i}^{*} \leq w_{j}^{*}$ for $1 \leq i<j \leq n$.

In what follows, we will construct a generalized version of Propositions 4.8 and 4.10.

Proposition 4.11. Suppose that $\boldsymbol{X}$ has an AI joint density. Then we have $w_{i}^{*} \leq w_{j}^{*}$ for any $1 \leq i<j \leq n$.

Proof. Since $\sum_{i=1}^{n} w_{i} x_{i}$ is AI on $(\boldsymbol{x}, \boldsymbol{w})$, by Theorem 3.1, we have, for any $1 \leq i<j \leq n$ and $w_{i} \leq w_{j}$,

$$
\sum_{k=1}^{n} w_{k} X_{k} \geq_{\mathrm{st}} w_{j} X_{i}+w_{i} X_{j}+\sum_{k \notin\{i, j\}} w_{k} X_{k}
$$

This implies that $w_{i}^{*} \leq w_{j}^{*}$ for any $1 \leq i<j \leq n$.

Evidently, Proposition 4.11 successfully extends Proposition 4.8. According to the proof of Theorem 5 in Li and You (2014), $s_{j}(x) / s_{i}(x)$ increases in $x \in\left(l_{i j}, u_{i j}\right)$ for any $1 \leq i<j \leq n$, and then the conditions of Proposition 4.10 fall into the setup of Theorem 9 of Li and You (2014). So, $\left(X_{1}, \ldots, X_{n}\right)$ in Proposition 4.10 has an AI joint density. That is, Proposition 4.11 covers Proposition 4.10 .

Next, we present an improved version of Proposition 4.9, which also eliminates the differentiability of the utility functions.

Proposition 4.12. Assume that $u$ is concave and $X$ has an Archimedean copula with a logconvex generator. Then $X_{i} \geq_{\mathrm{rh}} X_{j}$ implies that $w_{i}^{*} \geq w_{j}^{*}$ for $1 \leq i, j \leq n$.

Proof. It is easy to verify that $g(\boldsymbol{x}, \boldsymbol{w})=\sum_{i=1}^{n} w_{i} x_{i}$ is AI on $(\boldsymbol{x}, \boldsymbol{w})$ and increases in $x_{j}$ for any $j=1, \cdots, n$. Note that

$$
g\left(\boldsymbol{x}, \tau_{i j}(\boldsymbol{w})\right)-g(\boldsymbol{x}, \boldsymbol{w})=\left(w_{i}-w_{j}\right) x_{j}+\left(w_{j}-w_{i}\right) x_{i}
$$

is increasing in $x_{j}$ for $w_{i} \geq w_{j}$, and $g(\boldsymbol{x}, \boldsymbol{w})$ satisfies the corresponding conditions in Theorem 3.3 for $w_{i} \geq w_{j}$. As a result, by Corollary 3.4, $X_{i} \geq_{\mathrm{rh}} X_{j}$ implies that

$$
\sum_{i=1}^{n} w_{i} X_{i} \geq_{\mathrm{icv}} w_{j} X_{i}+w_{i} X_{j}+\sum_{k \notin\{i, j\}} w_{k} X_{k} \quad \text { for } w_{i} \geq w_{j} \text { and } 1 \leq i, j \leq n .
$$

Thus, it follows that $w_{i}^{*} \geq w_{j}^{*}$ for $1 \leq i, j \leq n$. 


\subsection{Financial portfolio allocation based on a distortion risk measure}

A risk measure is simply a functional assigning of a nonnegative real number to a risk. For a distortion function $h:[0,1] \rightarrow[0,1]$, which is increasing and satisfies $h(0)=0, h(1)=1$, the so-called distortion risk measure $\rho_{h}(X)$ for a risk $X$ with survival function $\bar{F}$ is defined as

$$
\rho_{h}(X)=\int_{0}^{\infty} h(\bar{F}(t)) \mathrm{d} t-\int_{-\infty}^{0}[1-h(\bar{F}(t))] \mathrm{d} t .
$$

Distortion risk measures have several useful properties, e.g. positive homogeneity, translation invariance, and additivity for comonotonic risks. Some distortion risk measures preserve appropriate stochastic orders. For more details; see, Denuit et al. (2005) and Dhaene et al. (2006).

Lemma 4.1. (Denuit et al. (2005, Proposition 3.4.7).) For random variables $X$ and $Y$,

(a) $X \leq_{\mathrm{icx}} Y$ if and only if $\rho_{h}(X) \leq \rho_{h}(Y)$ for all concave distortion function $h$;

(b) $X \leq_{\mathrm{icv}} Y$ if and only if $\rho_{h}(X) \leq \rho_{h}(Y)$ for all convex distortion function $h$.

Let us reconsider the financial portfolio allocation based on the distortion measure. From the investors' viewpoint, a good allocation policy is to ensure that the potential return is as great as possible. In the frame of distorted expectation theory, the optimization problem boils down to

$$
\max _{\alpha \in \mathcal{A}_{\alpha}} \rho_{h}\left(\sum_{i=1}^{n} \alpha_{i} X_{i}\right) \text { for } \alpha>0,
$$

where $\rho_{h}$ is the risk measure with distortion function $h$. Let $\left(\alpha_{1}^{*}, \ldots, \alpha_{n}^{*}\right)$ be the optimal solution of the above optimization problem. By Lemma 4.1(b) and the proof of Proposition 4.12, we immediately reach the following proposition.

Proposition 4.13. Suppose that the distortion function $h$ is convex and the concerned asset vector $\boldsymbol{X}$ has an Archimedean copula with a log-convex generator. Then $X_{i} \geq_{\mathrm{rh}} X_{j}$ implies that $\alpha_{i}^{*} \geq \alpha_{j}^{*}$ for $1 \leq i, j \leq n$.

As an application of Corollary 3.2, we also obtain the following result.

Proposition 4.14. Assume the distortion function $h$ is concave and $X$ has an Archimedean survival copula with a log-convex generator. Then $X_{i} \geq_{\mathrm{hr}} X_{j}$ implies that $\alpha_{i}^{*} \geq \alpha_{j}^{*}$ for $1 \leq$ $i, j \leq n$.

Proof. Let $g(\boldsymbol{x}, \boldsymbol{\alpha}):=\sum_{i=1}^{n} \alpha_{i} x_{i}$. It is easy to check that both $g(\boldsymbol{x}, \boldsymbol{\alpha})$ and

$$
g(\boldsymbol{x}, \boldsymbol{\alpha})-g\left(\boldsymbol{x}, \tau_{i j}(\boldsymbol{\alpha})\right)=\left(\alpha_{i}-\alpha_{j}\right) x_{i}-\left(\alpha_{i}-\alpha_{j}\right) x_{j}
$$

are increasing in $x_{i}$ for $\alpha_{i} \geq \alpha_{j}$. That is, $g(\boldsymbol{x}, \boldsymbol{\alpha})$ satisfies the related conditions of Theorem 3.2 for $\alpha_{i} \geq \alpha_{j}$. Consequently, as a result of Corollary 3.2, $X_{i} \geq_{\mathrm{hr}} X_{j}$ implies that, for $\alpha_{i} \geq \alpha_{j}$ and $1 \leq i, j \leq n$,

$$
\sum_{k=1}^{n} \alpha_{k} X_{k} \geq_{\text {icx }} \alpha_{j} X_{i}+\alpha_{i} X_{j}+\sum_{k \notin\{i, j\}}^{n} \alpha_{k} X_{k}
$$


From Lemma 4.1(a), it follows that, for any concave distortion function $h$,

$$
\rho_{h}\left(\sum_{k=1}^{n} \alpha_{k} X_{k}\right) \geq \rho_{h}\left(\alpha_{j} X_{i}+\alpha_{i} X_{j}+\sum_{k \notin\{i, j\}}^{n} \alpha_{k} X_{k}\right),
$$

and this implies that $\alpha_{i}^{*} \geq \alpha_{j}^{*}$ for $1 \leq i, j \leq n$.

\section{Appendix A. Proof of Theorem 3.2}

Note that the joint density $f(\boldsymbol{x})$ of the random vector $\boldsymbol{X}$ is UTPD or UTPI with respect to a pair $(i, j)$ with $i>j$ or $i<j$, that is,

$$
\int_{t}^{\infty}\left[f(\boldsymbol{x})-f\left(\tau_{i j}(\boldsymbol{x})\right)\right] \mathrm{d} x_{i} \geq 0 \quad \text { for } t \geq x_{j} \text { and } 1 \leq i, j \leq n .
$$

For any increasing convex function $u$ and the pair $(i, j)$, fixed $x_{k}$ with $k \neq i$, let $g_{1}\left(x_{i}\right)=$ $g(\boldsymbol{x}, \boldsymbol{\lambda})$ and $g_{2}\left(x_{i}\right)=g\left(\boldsymbol{x}, \tau_{i j}(\boldsymbol{\lambda})\right)$. By the increasing property of $g(\boldsymbol{x}, \boldsymbol{\lambda})-g\left(\boldsymbol{x}, \tau_{i j}(\boldsymbol{\lambda})\right)$, it follows that, for $y \geq x \geq x_{j}$ and $\lambda_{i} \geq \lambda_{j}, g_{1}(y)-g_{2}(y) \geq g_{1}(x)-g_{2}(x)$ and, hence,

$$
u\left(g_{1}(y)-\left[g_{1}(x)-g_{2}(x)\right]\right)-u\left(g_{2}(y)\right) \geq 0 .
$$

Note that the componentwise increasing property of $g(x, \lambda)$ guarantees that $g_{1}(y) \geq g_{1}(x)$ for $y \geq x \geq x_{j}$. On the other hand, since $g(\boldsymbol{x}, \lambda)$ is AI, it follows that, for $x_{i} \geq x_{j}$ and $\lambda_{i} \geq \lambda_{j}$,

$$
g_{1}\left(x_{i}\right)-g_{2}\left(x_{i}\right)=g(\boldsymbol{x}, \boldsymbol{\lambda})-g\left(\boldsymbol{x}, \tau_{i j}(\boldsymbol{\lambda})\right) \geq 0 .
$$

Thus, from the convexity of $u$, it follows that, for $y \geq x \geq x_{j}$,

$$
u\left(g_{1}(y)\right)-u\left(g_{1}(y)-\left[g_{1}(x)-g_{2}(x)\right]\right) \geq u\left(g_{1}(x)\right)-u\left(g_{2}(x)\right) .
$$

From (A.2) and (A.3), it follows that, for $y \geq x \geq x_{j}$,

$$
\begin{aligned}
& u\left(g_{1}(y)\right)-u\left(g_{2}(y)\right) \\
& \quad=u\left(g_{1}(y)\right)-u\left(g_{1}(y)-\left(g_{1}(x)-g_{2}(x)\right)\right)+u\left(g_{1}(y)-\left(g_{1}(x)-g_{2}(x)\right)\right)-u\left(g_{2}(y)\right) \\
& \quad \geq u\left(g_{1}(x)\right)-u\left(g_{2}(x)\right) .
\end{aligned}
$$

That is, for $\lambda_{i} \geq \lambda_{j}$,

$$
h\left(x_{i}\right)=\left[u\left(g_{1}\left(x_{i}\right)\right)-u\left(g_{2}\left(x_{i}\right)\right)\right] \mathbf{1}\left(x_{i} \geq x_{j}\right)=\left[u(g(\boldsymbol{x}, \boldsymbol{\lambda}))-u\left(g\left(\boldsymbol{x}, \tau_{i j}(\boldsymbol{\lambda})\right)\right] \mathbf{1}\left(x_{i} \geq x_{j}\right)\right.
$$

is nonnegative and increasing in $x_{i}$.

For fixed $x_{k}$ with $k \neq i$ and the pair $(i, j)$, let $w\left(x_{i}\right)=\left[f(\boldsymbol{x})-f\left(\tau_{i j}(\boldsymbol{x})\right)\right] \mathbf{1}\left(x_{i} \geq x_{j}\right)$. In view of (A.1), it follows that, for all $t$,

$$
\int_{t}^{\infty} w\left(x_{i}\right) \mathrm{d} x_{i}=\int_{t \vee x_{j}}^{\infty}\left[f(\boldsymbol{x})-f\left(\tau_{i j}(\boldsymbol{x})\right)\right] \mathrm{d} x_{i} \geq 0 .
$$

From Lemma 7.1(a) of Barlow and Proschan (1975), we immediately obtain, for $(i, j)$ with $\lambda_{i} \geq \lambda_{j}$,

$$
\begin{aligned}
\int_{x_{j}}^{\infty}\left[u(g(\boldsymbol{x}, \boldsymbol{\lambda}))-u\left(g\left(\tau_{i j}(\boldsymbol{x}), \lambda\right)\right)\right]\left[f(\boldsymbol{x})-f\left(\tau_{i j}(\boldsymbol{x})\right)\right] \mathrm{d} x_{i} & =\int_{-\infty}^{+\infty} h\left(x_{i}\right) w\left(x_{i}\right) \mathrm{d} x_{i} \\
& \geq 0
\end{aligned}
$$


As a result of the AI property of $g(\boldsymbol{x}, \lambda)$, it holds that $g(\boldsymbol{x}, \lambda)=g\left(\tau_{i j}(\boldsymbol{x}), \tau_{i j}(\boldsymbol{\lambda})\right)$ and $g\left(\tau_{i j}(\boldsymbol{x}), \lambda\right)=g\left(\boldsymbol{x}, \tau_{i j}(\lambda)\right)$. Thus, for any increasing and convex function $u$,

$$
\begin{aligned}
\mathbb{E}[u(g(\boldsymbol{X}, \boldsymbol{\lambda}))]-\mathbb{E}\left[u\left(g\left(\boldsymbol{X}, \tau_{i j}(\boldsymbol{\lambda})\right)\right)\right] & \int_{x_{i} \geq x_{j}, \mathbb{R}^{n-2}} u(g(\boldsymbol{x}, \boldsymbol{\lambda})) f(\boldsymbol{x}) \prod_{k=1}^{n} \mathrm{~d} x_{k}+\int_{x_{i} \leq x_{j}, \mathbb{R}^{n-2}} u(g(\boldsymbol{x}, \boldsymbol{\lambda})) f(\boldsymbol{x}) \prod_{k=1}^{n} \mathrm{~d} x_{k} \\
& -\int_{x_{i} \geq x_{j}, \mathbb{R}^{n-2}} u\left(g\left(\boldsymbol{x}, \tau_{i j}(\boldsymbol{\lambda})\right)\right) f(\boldsymbol{x}) \prod_{i=1}^{n} \mathrm{~d} x_{k}-\int_{x_{i} \leq x_{j}, \mathbb{R}^{n-2}} u\left(g\left(\boldsymbol{x}, \tau_{i j}(\boldsymbol{\lambda})\right)\right) f(\boldsymbol{x}) \prod_{k=1}^{n} \mathrm{~d} x_{k} \\
= & \int_{x_{i} \geq x_{j}, \mathbb{R}^{n-2}} u\left(g(\boldsymbol{x}, \boldsymbol{\lambda}) f(\boldsymbol{x}) \prod_{k=1}^{n} \mathrm{~d} x_{k}+\int_{x_{i} \geq x_{j}, \mathbb{R}^{n-2}}^{n} u\left(g\left(\tau_{i j}(\boldsymbol{x}), \boldsymbol{\lambda}\right)\right) f\left(\tau_{i j}(\boldsymbol{x})\right) \prod_{i=1}^{n} \mathrm{~d} x_{k}\right. \\
& -\int_{x_{i} \geq x_{j}, \mathbb{R}^{n-2}} u\left(g\left(\boldsymbol{x}, \tau_{i j}(\boldsymbol{\lambda})\right)\right) f(\boldsymbol{x}) \prod_{k=1}^{n} \mathrm{~d} x_{k} \\
& -\int_{x_{i} \geq x_{j}, \mathbb{R}^{n-2}} u\left(g\left(\tau_{i j}(\boldsymbol{x}), \tau_{i j}(\boldsymbol{\lambda})\right)\right) f\left(\tau_{i j}(\boldsymbol{x})\right) \prod_{k=1}^{n} \mathrm{~d} x_{k} \\
= & \int_{\mathbb{R}^{n-2}} \int_{x_{i} \geq x_{j}}\left[u(g(\boldsymbol{x}, \boldsymbol{\lambda}))-u\left(g(\boldsymbol{x}), \tau_{i j}(\boldsymbol{\lambda})\right)\right]\left[f(\boldsymbol{x})-f\left(\tau_{i j}(\boldsymbol{x})\right)\right] \mathrm{d} x_{i} \mathrm{~d} x_{j} \prod_{k \notin\{i, j\}} \mathrm{d} x_{k} \\
= & \int_{\mathbb{R}^{n-1}} \int_{x_{j}}^{\infty}\left[u(g(\boldsymbol{x}, \boldsymbol{\lambda}))-u\left(g(\boldsymbol{x}), \tau_{i j}(\boldsymbol{\lambda})\right)\right]\left[f(\boldsymbol{x})-f\left(\tau_{i j}(\boldsymbol{x})\right)\right] \mathrm{d} x_{i} \prod_{k \neq i} \mathrm{~d} x_{k} .
\end{aligned}
$$

Now, by virtue of (A.4), we conclude that the above $n$-fold integration is nonnegative and, hence, $\mathbb{E}[u(g(\boldsymbol{X}, \boldsymbol{\lambda}))] \geq \mathbb{E}\left[u\left(g\left(\boldsymbol{X}, \tau_{i j}(\boldsymbol{\lambda})\right)\right)\right]$ for $\lambda_{i} \geq \lambda_{j}$ and the pair $(i, j)$. That is just the desired increasing convex order.

The increasing convex order in the reverse direction may be constructed in a similar manner.

\section{Appendix B. Proof of Corollary 3.1}

Note that $\psi(0)=\infty$ and $s_{k}^{*}(x)=\psi^{(1)}\left(\bar{F}_{k}(x)\right) f_{k}(x)$, for $t \geq x_{j}$ and $1 \leq i, j \leq n$,

$$
\begin{aligned}
\int_{t}^{\infty}\left[f(\boldsymbol{x})-f\left(\tau_{i j}(\boldsymbol{x})\right)\right] \mathrm{d} x_{i} & \\
= & \int_{t}^{\infty} \prod_{k=1}^{n} s_{k}^{*}\left(x_{k}\right) \cdot \varphi^{(n)}\left(\sum_{k=1}^{n} \psi\left(\bar{F}_{k}\left(x_{k}\right)\right)\right) \mathrm{d} x_{i} \\
& -\int_{t}^{\infty} \prod_{k \notin\{i, j\}} s_{k}^{*}\left(x_{k}\right) \cdot s_{i}^{*}\left(x_{j}\right) s_{j}^{*}\left(x_{i}\right) \varphi^{(n)}\left(\psi\left(\bar{F}_{i}\left(x_{j}\right)\right)+\psi\left(\bar{F}_{j}\left(x_{i}\right)\right)\right. \\
& \left.+\sum_{k \notin\{i, j\}} \psi\left(\bar{F}_{k}\left(x_{k}\right)\right)\right) \mathrm{d} x_{i}
\end{aligned}
$$




$$
\begin{gathered}
=\prod_{k \notin\{i, j\}} s_{k}^{*}\left(x_{k}\right)\left\{s_{j}^{*}\left(x_{j}\right) \int_{t}^{\infty} \varphi^{(n)}\left(\sum_{k=1}^{n} \psi\left(\bar{F}_{k}\left(x_{k}\right)\right)\right) \mathrm{d}\left[-\psi\left(\bar{F}_{i}\left(x_{i}\right)\right)\right]\right. \\
-s_{i}^{*}\left(x_{j}\right) \int_{t}^{\infty} \varphi^{(n)}\left(\psi\left(\bar{F}_{i}\left(x_{j}\right)\right)+\psi\left(\bar{F}_{j}\left(x_{i}\right)\right)\right. \\
\left.\left.+\sum_{k \notin\{i, j\}} \psi\left(\bar{F}_{k}\left(x_{k}\right)\right)\right) \mathrm{d}\left[-\psi\left(\bar{F}_{j}\left(x_{i}\right)\right)\right]\right\} \\
=\prod_{k \notin\{i, j\}} s_{k}^{*}\left(x_{k}\right)\left\{s _ { j } ^ { * } ( x _ { j } ) \left[\varphi^{(n-1)}\left(\psi\left(\bar{F}_{i}(t)\right)+\psi\left(\bar{F}_{j}\left(x_{j}\right)\right)+\sum_{k \notin\{i, j\}} \psi\left(\bar{F}_{k}\left(x_{k}\right)\right)\right)\right.\right. \\
\left.-\lim _{s \rightarrow \infty} \varphi^{(n-1)}(s)\right]-s_{i}^{*}\left(x_{j}\right)\left[\varphi ^ { ( n - 1 ) } \left(\psi\left(\bar{F}_{i}\left(x_{j}\right)\right)+\psi\left(\bar{F}_{j}(t)\right)\right.\right. \\
\left.+\sum_{k \notin\{i, j\}} \psi\left(\bar{F}_{k}\left(x_{k}\right)\right)\right) \\
\left.\left.-\lim _{s \rightarrow \infty} \varphi^{(n-1)}(s)\right]\right\} \\
=\left[s_{j}^{*}\left(x_{j}\right) \Delta_{1}^{*}-s_{i}^{*}\left(x_{j}\right) \Delta_{2}^{*}\right] \prod_{k \notin\{i, j\}} s_{k}^{*}\left(x_{k}\right),
\end{gathered}
$$

where

$$
\begin{aligned}
& \Delta_{1}^{*}=\varphi^{(n-1)}\left(\psi\left(\bar{F}_{i}(t)\right)+\psi\left(\bar{F}_{j}\left(x_{j}\right)\right)+\sum_{k \notin\{i, j\}} \psi\left(\bar{F}_{k}\left(x_{k}\right)\right)\right)-\lim _{s \rightarrow \infty} \varphi^{(n-1)}(s), \\
& \Delta_{2}^{*}=\varphi^{(n-1)}\left(\psi\left(\bar{F}_{i}\left(x_{j}\right)\right)+\psi\left(\bar{F}_{j}(t)\right)+\sum_{k \notin\{i, j\}} \psi\left(\bar{F}_{k}\left(x_{k}\right)\right)\right)-\lim _{s \rightarrow \infty} \varphi^{(n-1)}(s) .
\end{aligned}
$$

As a result of $s_{i}^{*}(x) \geq s_{j}^{*}(x)$, it follows that, for any $t \geq x_{j}$,

$$
\begin{aligned}
0 & \leq \int_{x_{j}}^{t}\left(s_{i}^{*}(x)-s_{j}^{*}(x)\right) \mathrm{d} x \\
& =\int_{x_{j}}^{t}\left[\psi^{(1)}\left(\bar{F}_{i}(x)\right) f_{i}(x)-\psi^{(1)}\left(\bar{F}_{j}(x)\right) f_{j}(x)\right] \mathrm{d} x_{i} \\
& =\left[\psi\left(\bar{F}_{i}\left(x_{j}\right)\right)+\psi\left(\bar{F}_{j}(t)\right)\right]-\left[\psi\left(\bar{F}_{i}(t)\right)+\psi\left(\bar{F}_{j}\left(x_{j}\right)\right)\right] .
\end{aligned}
$$

Hence, the decreasing property of $(-1)^{n-1} \varphi^{(n-1)}$ implies that, for any $t \geq x_{j}$,

$$
\begin{aligned}
& (-1)^{n-1} \varphi^{(n-1)}\left(\psi\left(\bar{F}_{i}(t)\right)+\psi\left(\bar{F}_{j}\left(x_{j}\right)\right)+\sum_{k \notin\{i, j\}} \psi\left(\bar{F}_{k}\left(x_{k}\right)\right)\right) \\
& \geq(-1)^{n-1} \varphi^{(n-1)}\left(\psi\left(\bar{F}_{i}\left(x_{j}\right)\right)+\psi\left(\bar{F}_{j}(t)\right)+\sum_{k \notin\{i, j\}} \psi\left(\bar{F}_{k}\left(x_{k}\right)\right)\right) \\
& \geq \lim _{s \rightarrow \infty}(-1)^{n-1} \varphi^{(n-1)}(s)
\end{aligned}
$$


and, thus,

$$
\begin{aligned}
(-1)^{n-1} \Delta_{1}^{*}= & (-1)^{n-1} \varphi^{(n-1)}\left(\psi\left(\bar{F}_{i}(t)\right)+\psi\left(\bar{F}_{j}\left(x_{j}\right)\right)+\sum_{k \notin\{i, j\}} \psi\left(\bar{F}_{k}\left(x_{k}\right)\right)\right) \\
& -\lim _{s \rightarrow \infty}(-1)^{n-1} \varphi^{(n-1)}(s) \\
\geq & (-1)^{n-1} \varphi^{(n-1)}\left(\psi\left(\bar{F}_{i}\left(x_{j}\right)\right)+\psi\left(\bar{F}_{j}(t)\right)+\sum_{k \notin\{i, j\}} \psi\left(\bar{F}_{k}\left(x_{k}\right)\right)\right) \\
& -\lim _{s \rightarrow \infty}(-1)^{n-1} \varphi^{(n-1)}(s) \\
= & (-1)^{n-1} \Delta_{2}^{*} \\
\geq & 0 .
\end{aligned}
$$

Since $\psi(x)$ decreases, $s_{i}^{*}(x)=\psi^{(1)}\left(\bar{F}_{i}(x)\right) f_{i}(x) \leq 0$ for all $x$, and $s_{i}^{*}(x) \geq s_{j}^{*}(x)$ leads to $-s_{j}^{*}\left(x_{j}\right) \geq-s_{i}^{*}\left(x_{j}\right) \geq 0$. By (B.2), it follows that

$$
\begin{aligned}
(-1)^{n-2} s_{j}^{*}\left(x_{j}\right) \Delta_{1}^{*}-(-1)^{n-2} s_{i}^{*}\left(x_{j}\right) \Delta_{2}^{*} & =-s_{j}^{*}\left(x_{j}\right)(-1)^{n-1} \Delta_{1}^{*}-\left(-s_{i}^{*}\left(x_{j}\right)\right)(-1)^{n-1} \Delta_{2}^{*} \\
& \geq 0 .
\end{aligned}
$$

From (B.1) and $(-1)^{n-2} \prod_{k \notin\{i, j\}} s_{k}^{*}\left(x_{k}\right) \geq 0$, we may be conclude that

$$
\begin{aligned}
0 & \leq\left[-s_{j}^{*}\left(x_{j}\right)(-1)^{n-1} \Delta_{1}^{*}-\left(-s_{i}^{*}\left(x_{j}\right)\right)(-1)^{n-1} \Delta_{2}^{*}\right](-1)^{n-2} \prod_{k \notin\{i, j\}} s_{k}^{*}\left(x_{k}\right) \\
& =\int_{t}^{\infty}\left[f(\boldsymbol{x})-f\left(\tau_{i j}(\boldsymbol{x})\right)\right] \mathrm{d} x_{i} .
\end{aligned}
$$

This invokes (A.1) and, hence, completes the proof.

\section{Appendix C. Proof of Corollary 3.2}

Since the generator $\varphi$ is log-convex then $\varphi^{(1)}(x) / \varphi(x)$ is increasing, i.e. $\varphi(x) / \varphi^{(1)}(x)$ is decreasing. As a result of the decreasing property of $\psi=\varphi^{-1}$, it follows that $x \psi^{(1)}(x)=$ $\varphi(\psi(x)) / \varphi^{(1)}(\psi(x))$ is increasing. On the other hand, for $1 \leq i, j \leq n, X_{i} \geq_{\mathrm{hr}} X_{j}$ implies that $X_{i} \geq_{\text {st }} X_{j}$, i.e. $\bar{F}_{i}(x) \geq \bar{F}_{j}(x)$ for all $x$, and, hence, it holds that $\psi^{(1)}\left(\bar{F}_{i}(x)\right) \bar{F}_{i}(x) \geq$ $\psi^{(1)}\left(\bar{F}_{j}(x)\right) \bar{F}_{j}(x)$. In view of $\psi^{(1)}(x) \leq 0$, we obtain

$$
0 \leq \frac{\psi^{(1)}\left(\bar{F}_{i}(x)\right) \bar{F}_{i}(x)}{\psi^{(1)}\left(\bar{F}_{j}(x)\right) \bar{F}_{j}(x)} \leq 1 .
$$

Also, $X_{i} \geq$ hr $X_{j}$ implies that $f_{i}(x) / \bar{F}_{i}(x) \leq f_{j}(x) / \bar{F}_{j}(x)$ for all $x \geq 0$. As a result, we obtain

$$
\frac{\psi^{(1)}\left(\bar{F}_{i}(x)\right) \bar{F}_{i}(x)}{\psi^{(1)}\left(\bar{F}_{j}(x)\right) \bar{F}_{j}(x)} \frac{f_{i}(x)}{\bar{F}_{i}(x)} \leq \frac{f_{j}(x)}{\bar{F}_{j}(x)} .
$$

This results in

$$
s_{i}^{*}(x)=\psi^{(1)}\left(\bar{F}_{i}(x)\right) f_{i}(x) \geq \psi^{(1)}\left(\bar{F}_{j}(x)\right) f_{j}(x)=s_{j}^{*}(x) .
$$

Thus, by Corollary 3.1, we complete the proof. 


\section{Appendix D. Proof of Theorem 3.3}

Note that the joint density $f(\boldsymbol{x})$ of the random vector $\boldsymbol{X}$ is LTPD or LTPI with respect to a pair $(i, j)$ with $i>j$ or $i<j$, that is,

$$
\int_{-\infty}^{t}\left[f(\boldsymbol{x})-f\left(\tau_{i j}(\boldsymbol{x})\right)\right] \mathrm{d} x_{j} \geq 0 \text { for } t \leq x_{i} \text { and } 1 \leq i, j \leq n .
$$

Assume that function $u$ is increasing and concave. Let

$$
g_{1}\left(x_{j}\right)=g(\boldsymbol{x}, \boldsymbol{\lambda}) \quad \text { and } \quad g_{2}\left(x_{j}\right)=g\left(\boldsymbol{x}, \tau_{i j}(\boldsymbol{\lambda})\right) .
$$

Since $g(\boldsymbol{x}, \lambda)-g\left(\tau_{i j}(\boldsymbol{x}), \lambda\right)$ decreases in $x_{j}$ for $x_{j} \leq x_{i}$ and $\lambda_{i} \geq \lambda_{j}$, it holds that

$$
g_{1}(x)-g_{2}(x) \geq g_{1}(y)-g_{2}(y) \text { for } x \leq y \leq x_{i},
$$

and, hence,

$$
u\left(g_{1}(x)-\left(g_{1}(y)-g_{2}(y)\right)\right)-u\left(g_{2}(x)\right) \geq 0 \text { for } x \leq y \leq x_{i} .
$$

Since $g(\boldsymbol{x}, \lambda)$ is AI, it follows that, for $x_{j} \leq x_{i}$ and $\lambda_{i} \geq \lambda_{j}$,

$$
g_{1}\left(x_{j}\right)-g_{2}\left(x_{j}\right)=g(\boldsymbol{x}, \lambda)-g\left(\boldsymbol{x}, \tau_{i j}(\boldsymbol{\lambda})\right) \geq 0 .
$$

The increasing property of $g(\boldsymbol{x}, \lambda)$ implies that $g_{1}(y) \geq g_{1}(x)$ for $x \leq y \leq x_{i}$. As a result of the concavity of $u$, it follows that, for $x \leq y \leq x_{i}$,

$$
u\left(g_{1}(x)\right)-u\left(g_{1}(x)-\left(g_{1}(y)-g_{2}(y)\right)\right) \geq u\left(g_{1}(y)\right)-u\left(g_{2}(y)\right) .
$$

From (D.2) and (D.3), it follows that, for $x \leq y \leq x_{i}$,

$$
\begin{aligned}
& u\left(g_{1}(x)\right)-u\left(g_{2}(x)\right) \\
& \quad=u\left(g_{1}(x)\right)-u\left(g_{1}(x)-\left(g_{1}(y)-g_{2}(y)\right)\right)+u\left(g_{1}(x)-\left(g_{1}(y)-g_{2}(y)\right)\right)-u\left(g_{2}(x)\right) \\
& \quad \geq u\left(g_{1}(y)\right)-u\left(g_{2}(y)\right) .
\end{aligned}
$$

That is,

$$
h\left(x_{j}\right)=\left[u(g(\boldsymbol{x}, \lambda))-u\left(g(\boldsymbol{x}), \tau_{i j}(\boldsymbol{\lambda})\right)\right] \mathbf{1}\left(x_{j} \leq x_{i}\right) \geq 0
$$

is decreasing in $x_{j}$ for $\lambda_{i} \geq \lambda_{j}$.

Let $w\left(x_{j}\right)=\left[f(\boldsymbol{x})-f\left(\tau_{i j}(\boldsymbol{x})\right)\right] \mathbf{1}\left(x_{j} \leq x_{i}\right)$. By virtue of (D.1), we obtain

$$
\int_{-\infty}^{t} w\left(x_{j}\right) \mathrm{d} x_{j}=\int_{-\infty}^{t \wedge x_{i}}\left[f(\boldsymbol{x})-f\left(\tau_{i j}(\boldsymbol{x})\right)\right] \mathrm{d} x_{j} \geq 0,
$$

for all $t$ and $1 \leq i, j \leq n$. According to Lemma 7.1(b) of Barlow and Proschan (1981), it holds that, for $\lambda_{i} \geq \lambda_{j}$ and the pair $(i, j)$,

$$
\int_{-\infty}^{x_{i}}\left[u(g(\boldsymbol{x}, \lambda))-u\left(g\left(\tau_{i j}(\boldsymbol{x}), \boldsymbol{\lambda}\right)\right)\right]\left[f(\boldsymbol{x})-f\left(\tau_{i j}(\boldsymbol{x})\right)\right] \mathrm{d} x_{j}=\int_{-\infty}^{\infty} h\left(x_{j}\right) w\left(x_{j}\right) \mathrm{d} x_{j} \geq 0 .
$$

Now, in a similar manner to the proof of Theorem 3.2, it follows that, for $\lambda_{i} \geq \lambda_{j}$ and the pair $(i, j)$,

$$
\begin{aligned}
0 & \leq \int_{\mathbb{R}^{n-1}} \int_{-\infty}^{x_{i}}\left[u(g(\boldsymbol{x}, \lambda))-u\left(g(\boldsymbol{x}), \tau_{i j}(\boldsymbol{\lambda})\right)\right]\left[f(\boldsymbol{x})-f\left(\tau_{i j}(\boldsymbol{x})\right)\right] \mathrm{d} x_{j} \prod_{k \neq j} \mathrm{~d} x_{k} \\
& =\mathbb{E}[u(g(\boldsymbol{X}, \boldsymbol{\lambda}))]-\mathbb{E}\left[u\left(g\left(\boldsymbol{X}, \tau_{i j}(\boldsymbol{\lambda})\right)\right)\right] .
\end{aligned}
$$

That is, $\mathbb{E}[u(g(\boldsymbol{X}, \boldsymbol{\lambda}))] \geq \mathbb{E}\left[u\left(g\left(\boldsymbol{X}, \tau_{i j}(\boldsymbol{\lambda})\right)\right)\right]$, yielding the desired increasing concave order.

The order in the reverse direction may be constructed in a similar manner. 


\section{Appendix E. Proof of Corollary 3.3}

Since $\psi(0)=\infty$ and $s_{k}(x)=\psi^{(1)}\left(F_{k}(x)\right) f_{k}(x)$, it holds that

$$
\begin{aligned}
\int_{-\infty}^{t}\left[f(\boldsymbol{x})-f\left(\tau_{i j}(\boldsymbol{x})\right)\right] \mathrm{d} x_{j} & \\
= & \int_{-\infty}^{t} \prod_{k=1}^{n} s_{k}\left(x_{k}\right) \varphi^{(n)}\left(\sum_{k=1}^{n} \psi\left(F_{k}\left(x_{k}\right)\right)\right) \mathrm{d} x_{j}
\end{aligned}
$$

for $t \leq x_{i}$ and $1 \leq i, j \leq n$, where

$$
\begin{aligned}
& \Delta_{1}=\varphi^{(n-1)}\left(\psi\left(F_{i}\left(x_{i}\right)\right)+\psi\left(F_{j}(t)\right)+\sum_{k \notin\{i, j\}} \psi\left(F_{k}\left(x_{k}\right)\right)\right)-\lim _{s \rightarrow \infty} \varphi^{(n-1)}(s), \\
& \Delta_{2}=\varphi^{(n-1)}\left(\psi\left(F_{i}(t)\right)+\psi\left(F_{j}\left(x_{i}\right)\right)+\sum_{k \notin\{i, j\}} \psi\left(F_{k}\left(x_{k}\right)\right)\right)-\lim _{s \rightarrow \infty} \varphi^{(n-1)}(s) .
\end{aligned}
$$

As a result of $s_{i}(x) \leq s_{j}(x)$, it follows that, for $t \leq x_{i}$,

$$
\begin{aligned}
0 & \geq \int_{t}^{x_{i}}\left(s_{i}(x)-s_{j}(x)\right) \mathrm{d} x \\
& =\int_{t}^{x_{i}}\left[\psi^{(1)}\left(F_{i}(x)\right) f_{i}(x)-\psi^{(1)}\left(F_{j}(x)\right) f_{j}(x)\right] \mathrm{d} x_{i} \\
& =\psi\left(F_{i}\left(x_{i}\right)\right)+\psi\left(F_{j}(t)\right)-\psi\left(F_{i}(t)\right)-\psi\left(F_{j}\left(x_{i}\right)\right) .
\end{aligned}
$$

That is, $\psi\left(F_{i}\left(x_{i}\right)\right)+\psi\left(F_{j}(t)\right) \leq \psi\left(F_{i}(t)\right)+\psi\left(F_{j}\left(x_{i}\right)\right)$.

Now, in a similar manner to the proof of Corollary 3.1, (D.1) may be invoked. And by Theorem 3.3, we complete the proof. 


\section{Appendix F. Proof of Corollary 3.4}

For each pair $(i, j)$, since $X_{i} \geq_{\text {rh }} X_{j}$ implies that $F_{i}(x) \leq F_{j}(x)$ and $f_{i}(x) / F_{i}(x) \geq$ $f_{j}(x) / F_{j}(x)$ for all $x \geq 0$, in a similar manner to the proof of Corollary 3.2, we can prove $s_{i}(x) \leq s_{j}(x)$ for all $x$ and $1 \leq i, j \leq n$. And, thus, the desired result immediately follows from Corollary 3.3.

\section{Acknowledgements}

This research was supported by the National Natural Science Foundation of China (11171278). The authors would like to thank the anonymous reviewer for his/her valuable comments on an earlier version of this manuscript. In particular, the application concerned with the distortion risk measure in Subsection 4.4 is inspired by the pertinent suggestion from the reviewer.

\section{References}

Barlow, R. E. And Proschan, F. (1975). Statistical Theory of Reliability and Life Testing: Probability Models. Holt, Rinehart and Winston, New York.

Boland, P. J. AND Proschan, F. (1988). Multivariate arrangement increasing functions with applications in probability and statistics. J. Multivariate Anal. 25, 286-298.

Boland, P. J., Proschan, F. And Tong, Y. L. (1988). Moment and geometric probability inqualities arising from arrangement increasing functions. Ann. Prob. 16, 407-413.

Chen, Z. And Hu, T. (2008). Asset proportions in optimal portfolios with dependent default risks. Insurance Math. Econom. 43, 223-226.

Cheung, K. C. (2006). Optimal portfolio problem with unknown dependency structure. Insurance Math. Econom. 38, 167-175.

Cheung, K. C. (2007). Optimal allocation of policy limits and deductibles. Insurance Math. Econom. 41, $382-391$.

CheUnG, K. C. AND YANG, H. (2004). Ordering optimal proportions in the asset allocation problem with dependent default risks. Insurance Math. Econom. 35, 595-609.

Denuit, M., Dhaene, J., Goovaerts, M. And KaAs, R. (2005). Actuarial Theory for Dependent Risks. John Wiley, Hoboken, NJ.

Dhaene, J., Tsanakas, A., Valdez, E. A. and Vanduffel, S. (2012). Optimal capital allocation principles. J. Risk Insurance 79, 1-28.

Dhaene, J. et al. (2006). Risk measures and comonotonicity: a review. Stoch. Models 22, 573-606.

Esary, J. D. And Proschan, F. (1972). Relationships among some concepts of bivariate dependence. Ann. Math. Statist. 43, 651-655.

Frostig, E., ZAKs, Y. AND LEviKson, B. (2007). Optimal pricing for a heterogeneous portfolio for a given risk factor and convex distance measure. Insurance Math. Econom. 40, 459-467.

Furman, E. AND Zitikis, R. (2008). Weighted risk capital allocations. Insurance Math. Econom. 43, $263-269$.

Genest, C. AND MacKay, J. (1986a). Copules archimédiennes et familles de lois bidimensionnelles dont les marges sont données. Canad. J. Statist. 14, 145-159.

Genest, C. And MacKay, J. (1986b). The joy of copulas: bivariate distributions with uniform marginals. Amer. Statistician 40, 280-283.

Genest, C. And Rivest, L.-P. (1993). Statistical inference procedures for bivariate Archimedean copulas. J. Amer. Statist. Assoc. 88, 1034-1043.

Hennessy, D. A. And Lapan, H. E. (2002). The use of Archimedean copulas to model portfolio allocations. Math. Finance 12, 143-154.

Hollander, M., Proschan, F. and Sethuraman, J. (1977). Functions decreasing in transportation and their applications in ranking problems. Ann. Statist. 5, 722-733.

HuA, L. AND CHEUnG, K. C. (2008a). Stochastic orders of scalar products with applications. Insurance Math. Econom. 42, 865-872.

Hua, L. And Cheung, K. C. (2008b). Worst allocations of policy limits and deductibles. Insurance Math. Econom. 43, 93-98.

Joe, H. (1997). Multivariate Models and Dependence Concepts. Chapman \& Hall, London.

KiJima, M. AND OHNishi, M. (1996). Portfolio selection problems via the bivariate characterization of stochastic dominance relations. Math. Finance 6, 237-277. 
Kimberling, C. H. (1974). A probabilistic interpretation of complete monotonicity. Aequationes Math. 10, 152-164.

Laeven, R. J. A. And Goovaerts, M. J. (2004). An optimization approach to the dynamic allocation of economic capital. Insurance Math. Econom. 35, 299-319.

LANDSBerger, M. And Meilijson, I. (1990). Demand for risky financial assets: a portfolio analysis. J. Econom. Theory 50, 204-213.

Landsman, Z. M. and Valdez, E. A. (2003). Tail conditional expectations for elliptical distributions. N. Amer. Actuarial J. 7, 55-71.

LI, H. AND LI, X. (eds) (2013). Stochastic Orders in Reliability and Risk. Springer, New York.

LI, X. AND You, Y. (2014). A note on allocation of portfolio shares of random assets with Archimedean copula. Ann. Operat. Res. 212, 155-167.

Lu, Z. Y. AND Meng, L. L. (2011). Stochastic comparisons for allocations of policy limits and deductibles with applications. Insurance Math. Econom. 48, 338-343.

Marshall, A. W. and Olkin, I. (1988). Families of multivariate distributions. J. Amer. Statist. Assoc. 83, 834-841.

Marshall, A. W., Olkin, I. And Arnold, B. C. (2011). Inequalities: Theory of Majorization and Its Applications, 2nd edn. Springer, New York.

McNeIL, A. J. AND NeŠLehovÁ, J. (2009). Multivariate Archimedean copulas, $d$-monotone functions and $l_{1}$-norm symmetric distributions. Ann. Statist. 37, 3059-3097.

McNeil, A. J., Frey, R. And Embrechts, P. (2005). Quantitative Risk Management. Princeton University Press.

Müller, A. And Stoyan, D. (2002). Comparison Methods for Stochastic Models and Risks. John Wiley, Chichester.

Myers, S. C. ANd ReAd, J. A., JR. (2001). Capital allocation for insurance companies. J. Risk Insurance 68, 545-580.

Nelsen, R. B. (2006). An Introduction to Copulas, 2nd edn. Springer, New York.

PANJer, H. H. (2001). Measurement of risk, solvency requirements, and allocation of capital within financial conglomerates. Res. Rep., Department of Statistics and Actuarial Science, University of Waterloo.

ShaKed, M. And Shanthikumar, J. G. (2007). Stochastic Orders. Springer, New York.

TsanaKas, A. (2004). Dynamic capital allocation with distortion risk measures. Insurance Math. Econom. 35, $223-243$.

TsanaKas, A. (2009). To split or not to split: capital allocation with convex risk measures. Insurance Math. Econom. 44, 268-277.

Xu, M. AND Hu, T. (2012). Stochastic comparisons of capital allocations with applications. Insurance Math. Econom. 50, 293-298.

Zhuang, W., Chen, Z. And Hu, T. (2009). Optimal allocation of policy limits and deductibles under distortion risk measures. Insurance Math. Econom. 44, 409-414. 